\title{
Atypical benign angioproliferative lesion in a cat: Diagnostic imaging pitfalls
}

\author{
ANNA ŁOJSZCZYK, ALEKSANDRA KIMICKA*, BARBARA LISIAK \\ Laboratory of Radiology and Ultrasonography, Department and Clinic of Animal Surgery, \\ *Department of Animal Physiology, Faculty of Veterinary Medicine, \\ University of Life Sciences in Lublin, Akademicka 13, 20-950 Lublin, Poland
}

Łojszczyk A., Kimicka A., Lisiak B.

\section{Atypical benign angioproliferative lesion in a cat: Diagnostic imaging pitfalls}

Summary

The authors present a case report of an atypical liver vascular malformation in a cat. The lesion was diagnosed first by ultrasound, fine needle aspiration and computed tomographic examination, which revealed the presence of a thin-walled single echogenic oval structure in the caudate lobe of the liver without visible flow in Doppler examination and with content similar to blood. The echogenicity of the lesion was variable, which depended on its size and time after aspiration. Initially, atypical haemangioma was suspected. After surgical removal, histopathological examinations were performed. The final diagnosis was a benign angioproliferative lesion.

Keywords: vascular liver malformation, benign angioproliferative lesion, haemangioma, ultrasound, computed tomography

Vascular anomalies are extensively discussed in medicine and veterinary medicine. In humans, the classification system divides vascular anomalies into primary categories: vasoproliferative or vascular neoplasm and vascular malformations (6). All structural abnormalities of the capillary, venous, lymphatic and arterial systems can be divided into slow-flow (capillary, venous and lymphatic) and fast-flow (arterial and arteriovenous) disorders $(6,9)$.

In animals, benign and malignant vascular neoplasms are extensively described, including mainly haemangiomas and angiosarcomas (3). More than half occur in the skin, the next most common sites being the spleen and liver (3).

Similarly, a broad spectrum of congenital and acquired anomalies have in recent years been detected in dogs and cats, especially affecting the portal venous system. This tendency is due to the growing availability of advanced imaging techniques in veterinary practice (2). Congenital disorders of the portal system reflect a variety of embryonic or foetal disturbances, which can be isolated or combined in complex vascular patterns and may lead to acquired vascular disorders (2). Many of them are differentiated on the basis of diagnostic imaging techniques, such as ultrasonography and computed tomography. The disorders most commonly mentioned in the veterinary literature are portosystemic shunts and arteriovenous fistulas. Other malformations, unlike in humans, are described much less often.

In the present paper, the authors present a case report of an atypical vascular liver malformation in a cat. To the best of our knowledge, this malformation should be classified in the category of slow-flow venous abnormalities. Similar cases are very rarely described in veterinary medicine.

\section{Case report}

A neutered mixed-breed male cat aged 4 years was presented at the clinic with clinical signs of vomiting and weakened appetite for two days. In addition to appetite and vomiting problems, apathy and irritability were reported in the clinical history. The cat had been found at the age of 5 weeks. It had a diaphragmatic hernia at that time and was operated on without postoperative complications. At the age of 11 months, triaditis was diagnosed.

The patient first underwent clinical testing. In the physical examination, the Body Condition Score (BCS) was rated 5, and the mucous membranes were pink and moist. It had full dentition, a normal bite and no dental plaque. The superficial lymph nodes were non-enlarged. During palpation, the abdomen was soft. The body temperature was within physiological limits.

After the clinical examination, blood was collected for morphological and biochemical examination, which did not show deviations from the norms for the given species. Next, the patient was referred for an abdominal ultrasound. 
The ultrasound examination was performed with a Mindray D7 using microconvex and linear probes (5-10 $\mathrm{MHz}$ ). The ultrasound revealed the presence of a single thin-walled anechogenic oval structure in the caudate lobe of the liver with dimensions of $3.05 \times 1.75 \times 1.5 \mathrm{~cm}$ without visible flow in the Doppler examination (Fig. 1). There were no visible abnormal vessels adjacent to the lesion at the site. The caudal vena cava and portal veins, as well as the aorta, had diameters within normal limits. The liver was normal in size with echogenicity and echostructure. The gallbladder was situated on the left side of the liver, and was thinwalled with echogenic content. The bile ducts were non-enlarged. The

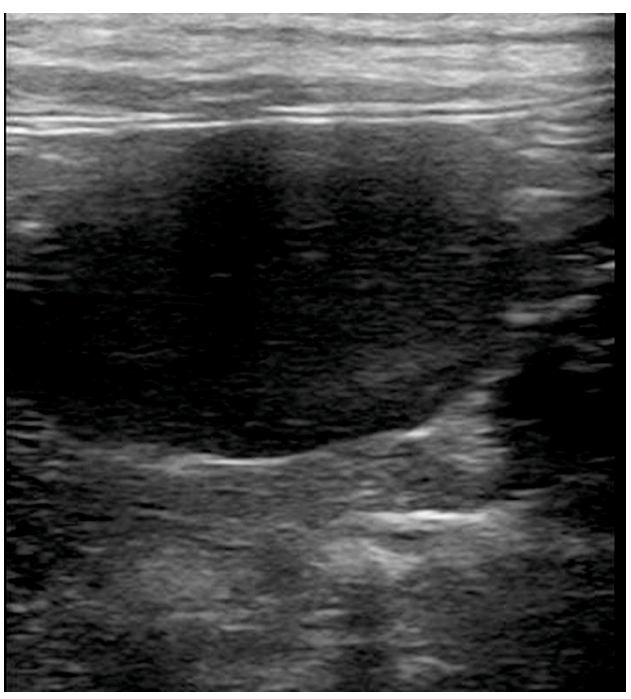

Fig. 1. The first ultrasound examination. A thin-walled lesion with aechogenic content is visible

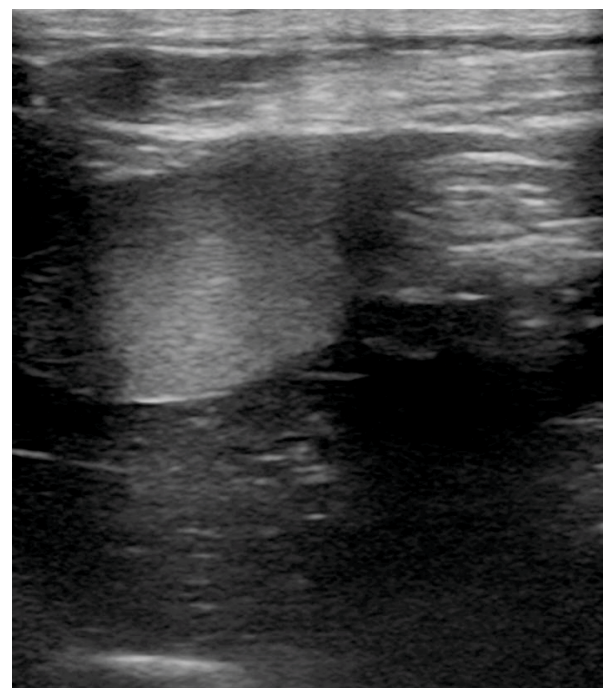

Fig. 2. The ultrasound examination after fine-needle aspiration. Hyperechoic content is visible within the lesion

jejunal lymph nodes were slightly enlarged and reactive. The other abdominal organs were within normal limits for cats.

After the ultrasound examination, fluid from the cyst was partially aspirated for further evaluation. The aspirate's colour was similar to blood. After fluid sampling, the followup examination revealed the same hyperechoic lesion, which showed visible internal fluid movement (Fig. 2). The cyst was $2.5 \times 1.5 \times 1.2 \mathrm{~cm}$ in size. There were no complications or fluid leakage after aspiration.

The fluid collected during biopsy was transferred for microbiological culture and cytological evaluation. The cytologic examination showed very numerous erythrocytes, as well as single neutrophils and lymphocytes. There were no cytological features suggesting a tumour-like lesion. No microbial growth was observed in the microbiological culture.

The initial diagnosis based on the above-mentioned examinations was atypical haemangioma, but to improve the evaluation, a computed tomographic examination was performed. The cat was anaesthetised with a $10 \mathrm{mg} / \mathrm{ml}$ dose of propofol (Scanofol, ScanVet, Poland) and a tomographic (CT) examination was performed with a Philips MX-16 slice scanner. Images were acquired with a soft algorithm in a soft tissue window that included unenhanced and contrast-enhanced phases with an IV injection of $2 \mathrm{ml} / \mathrm{kg}$ bw iohexol (Omnipaque $300 \mathrm{mgI} / \mathrm{ml}$, GE Healthcare AS, Oslo, Norway). The image acquisition parameters were $120 \mathrm{kV}$, $120 \mathrm{mAs} /$ slice, pitch 1.0069 , slice thickness $1.0 \mathrm{~mm}$, slice increment $0.5 \mathrm{~mm}$, collimation $16^{*} 0.75$ and rotation time $0.75 \mathrm{~s}$. Late arterial, early venous and venous phases were obtained. The images were sent to the Philips IntelliSpace Portal and to another computer unit, where they were analysed with the Horos 3.3.6 program.

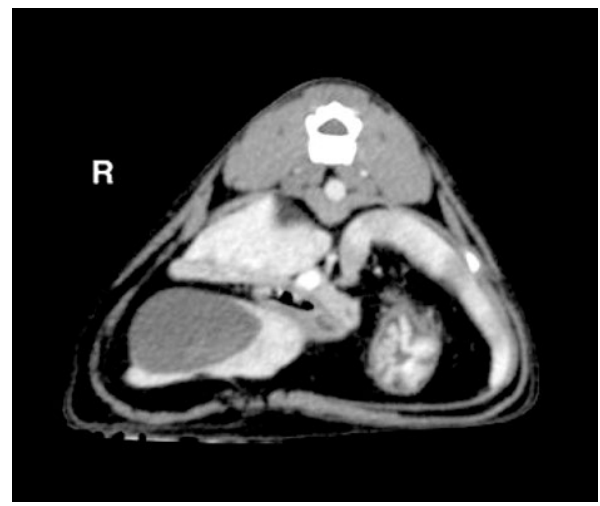

Fig. 4a. Computed tomography. Soft tissue algorithm. A computed tomography image of the lesion from figure 3 . On the ventral part of the lesion, there is a fluid-filled structure with a visible hyperdense region. There is no contrast enhancement inside or abnormal vessels adjacent to the lesion

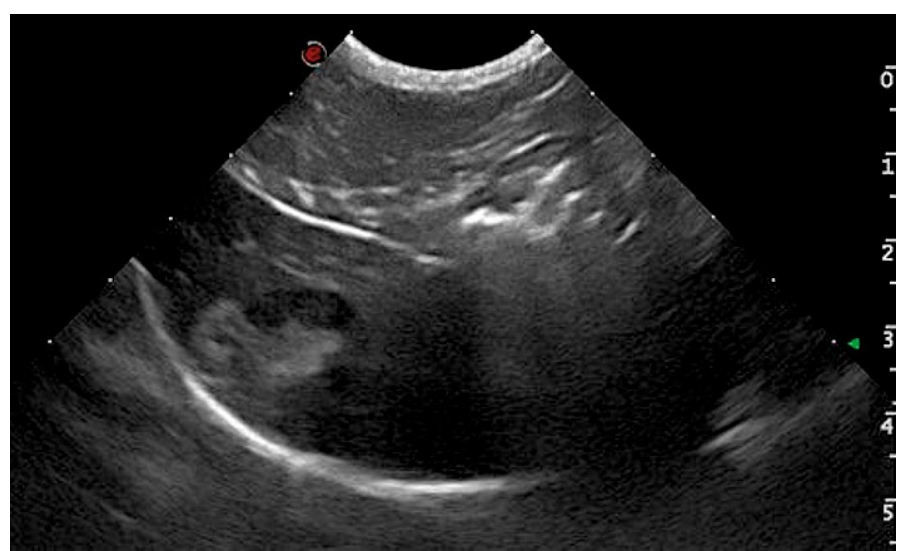

Fig. 3. Ultrasound examination at the time of computed tomography. Anechoic content with hyperechoic debris can be seen

The findings from the CT scan included the presence of a fluid-filled structure with attenuation of about $14 \mathrm{HU}$, which was observed previously in the ultrasound examination. The dimensions of the lesion were larger than previ-

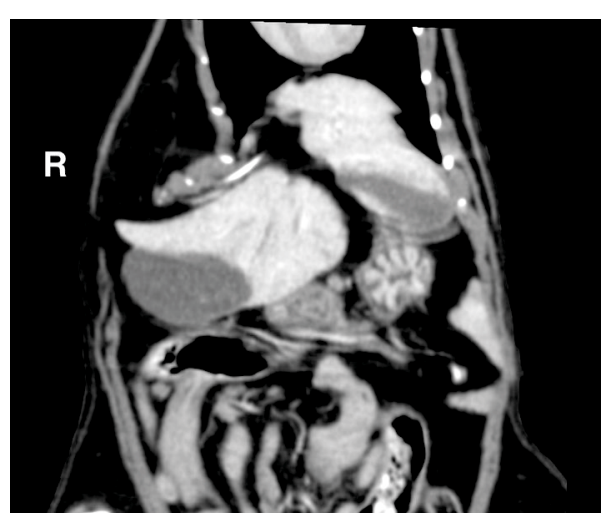

Fig. 4b. Computed tomography. Soft tissue algorithm. The malformation is visible on the right in the region of the caudate liver lobe. The gallbladder is situated to the left of the liver 
ously observed $(3.4 \times 2.3 \times 2.2 \mathrm{~cm})$. A hyperdense region of about $1.6 \times 1 \mathrm{~cm}$ with attenuation of $45 \mathrm{HU}$ was visible in the peripheral middle part of the structure. There were no contrast-enhancing areas in the lesion. The portal veins and the veins from the vena cava adjacent to the lesion were straight and not visibly enlarged. The gallbladder was situated in the area of the left lateral lobe and was filled with bile with attenuation of 34 HU. The bile ducts were not visible.

At the same time as the CT scan, a follow-up ultrasound examination was performed on an Easaote MyLab Twice ultrasound unit. The lesion was anechoic with the presence of a hyperechoic sedimentation layer. Based on the test results and the patient's clinical condition, the decision was made to perform surgery. After removal, the fragment of the liver lobe with the cyst was sent for histopathological evaluation (IDEXX laboratory), which showed the preserved lobular architecture of the liver and fibrosis with mild-tomoderate mononuclear-to-mixed inflammation, as well as mild bile duct proliferation in the portal fields. The large cyst in the centre of the sample was septated by fibrin and partially filled with blood, and it was surrounded by dilated vessels with mild fibrosis and haemorrhages, forming a kind of capsule. The final diagnosis was an angioproliferative benign lesion.

After the surgical intervention, supplementation with Hepaxan cat Vebiot (Nutrifarm, Dębica, Poland) was introduced at a dose of half a pill daily. Intravenous infusions of Ringer's fluid at a dose of $50 \mathrm{ml}$ per $\mathrm{kg}$ body weight were administered for 3 days after surgery, and intravenous maropitant at a dose of $1 \mathrm{mg} / \mathrm{kg}$ body weight for 4 days. For 3 days after surgery, buprenorphine was used intramuscularly at a dose of $0.02 \mathrm{mg}$ per $\mathrm{kg}$ body weight every eight hours. One month after the surgery, the owners reported an improvement in the general condition of the animal and did not observe any concerning symptoms.

Two weeks after the surgery, blood tests were performed. All parameters were normal, with the exception of platelets $73210^{\wedge} 3 / \mathrm{uL}\left(100-40010^{\wedge} 3 / \mathrm{ulL}\right)$, alanine aminotransferase $309 \mathrm{U} / 1$ (20-107 U/1), aspartate transaminase $74 \mathrm{U} / 1$ (6-44 $\mathrm{U} / 1$ ) and alkaline phosphatase $15 \mathrm{U} / 1$ (23-107 U/1).

Another blood test was performed four weeks after surgery, and the results were normal.

\section{Discussion}

The present case was not easy to interpret on the basis of diagnostic imaging. Upon histopathological examination, the removed liver cyst was classified as a benign vascular malformation, but atypical haemangioma was initially suspected. Haemangiomas are benign neoplastic lesions of blood vessels, usually asymptomatic and found accidentally (5). They are the most common benign tumours of the liver, consisting of blood-filled cavities lined by endothelial cells and fed by the hepatic artery $(1,11)$. The most common location, as in our patient, is the right liver lobe, and they are often marginal (1). The sonographic appearance of a haemangioma depends on its size, and these lesions may change echogenicity with a change in decubitus (7). The typical appearance of a haemangioma in an ultrasound is nodular hyperechoic and homogenous with well-defined margins and distal acoustic enhancement, but a larger lesion could have an inhomogeneous pattern $(1,11)$. This could be an effect of necrosis of the foci, haemorrhage or fibrosis, as well as the presence of stagnant blood, which could cause a sedimentation effect that is also visible on a CT scan $(1,11)$. In these cases, the superior fluid layer consists of unclotted serous blood and the inferior layer is red blood cells (11). An atypical appearance of a haemangioma in medicine could lead to misinterpretation (11). Doppler ultrasound may not detect any changes, because of the low velocity of the blood flow in these lesions $(1,7)$. In these cases, a CT examination is the method of choice to differentiate liver haemangiomas from other malformations.

In the present case, the ultrasound view was very similar to what was described above. The echogenicity of the lesion was variable, depending on its size and time after aspiration. The lesion was initially anechoic. After aspiration, the echogenicity changed to hyperechoic, with a lot of internal morphotic elements. At the time of the CT examination, the lesion was anechoic with the presence of a hyperechoic sedimentation layer, which suggests that stagnant blood could be observed in the lesion. There was no visible flow signal in the Doppler examination, but the lesion increased some time after aspiration.

The CT examination did not confirm the initial diagnosis. On CT scans, haemangiomas are typically hypodense and well-defined $(1,11)$. After the administration of contrast, in the arterial phase, an early rapid peripheral nodular enhancement is visible, with similar attenuation to the aorta $(1,11)$. In the later phase, there is visible progressive centripetal homogeneous filling, all the lesion is filled by contrast and could be isoattenuating to the liver. This feature is very suggestive of haemangioma $(1,11)$. In large lesions, the appearance on a CT scan could be heterogeneous with globular peripheral enhancements still visible (11). These typical features were absent in our case. All lesions were hypodense and aseptate, without any contrast enhancement, with a typical smooth, thin-walled appearance, which is rather typical of a simple liver cyst (8). There was also no visible abnormal vascularisation. The lesion was removed, and the final diagnosis was based on histopathological examination.

Vascular angioproliferative lesions are not frequently described in the veterinary literature. Reports describe mostly portosystemic shunts and arteriovenous fistulas. The histopathological characteristics of our lesion were similar to the enlargement of the aneurysmal vein, which is frequently reported in arteriovenous fistulas (9). Due to chronic portal hypertension and turbulent arterial flow, veins could dilate, with a weakening of the vessel wall and a progressive thickening of the intima and replacement by fibrous tissue (9). Gallbladder 
and bile duct abnormalities are also often described in these cases (9).

There were no signs of portal hypertension, such as pancreatic and gastric wall oedema or ascites, in our case, but we did not measure the portal flow velocity during the ultrasound examination (9). The portal pressure was not measured either. When arteriovenous fistulas or other venous abnormalities are suspected, selective dual-phase angiography with a time-attenuation contrast curve in the aorta, vena cava and portal vein is an important diagnostic tool $(12,13)$. We had only late arterial, early venous and venous phases, and the limitations of our equipment prevented the measurement of the timing and attenuation of the contrast bolus. On the other hand, we did not observe any diagnostic imaging signs that are described in arteriovenous fistulas in the ultrasound or computed tomography scan. The most common in these cases are abnormal arterioportal communications, enlargement of the afferent artery, a decreased diameter of the caudal aorta caudal to the afferent artery and microhepatia with intrahepatic duct mineralisation (9). In addition, contrast filling should be visible in aneurysmal vessels, and all arteriovenous fistulas are hypervascular fast-flow abnormalities (4, 6,12 ). In our case, no contrast filling was observed, and there was no visible flow in any part of the lesion in the Doppler examination, which precludes the possibility of an aneurysm in this location.

A similar lack of contrast filling is possible in structural vascular lesions which are slow-flow abnormalities. They are observed in humans and have a very similar appearance to liver haemangiomas in adults, which results in many false diagnoses (6).

Slow-flow abnormalities could arise from the venous, capillary or lymphatic system, or a combination thereof (6). These malformations contain abnormally formed and dilated superficial or deep veins, the walls of which are thin because they lack smooth muscle or are formed of collections of lymph vessels filled with serous fluid (6). In diagnostic imaging, they could be cystic, with or without a fluid and debris level (mostly lymphatic) and solid (mostly venous) components (6).

There are only a few publications on slow-flow abnormalities described above in the medicine and veterinary literature. Our diagnostic imaging findings, that is, the appearance of the cyst with a timedependent change in lesion echogenicity, the presence of a debris level, indicating that the blood inside the lesion is stagnant, the enlargement of the lesion for a time after aspiration, the absence of visible flow inside the lesion and the absence of visible abnormal vascularisation all suggest that our case is that of a vascular malformation which could be a slow-flow vascular capillary malformation. Histological results in these cases are decisive after treatment, which is the resection of the affected liver lobes (10). In the future, this topic should be analysed more broadly on the basis of a larger number of similar cases.

\section{References}

1. Bajenaru N., Savulescu F., Campenau I., Patrascu T.: Hepatic hemangioma. J. Med. Life 2015, 8, 4-11.

2.Bertolini G.: Acquired portal collateral circulation in the dog and cat. Vet. Radiol. Ultrasound 2010, 51, 25-33.

3. Gamlen H., Nordstoga K., Arnesen K.: Canine vascular neoplasia - a population based clinicopathologic study of 439 tumours and tumours like lesions in 420 dogs. APMIS Suppl. 2008, 125, 41-54.

4. Koc Z., Oguzkurt L., Ulusan S.: Portal venous system aneurysm: imaging, clinical findings, and a possible new etiologic factor. AJR 2007, 189, 1023 -1029 .

5. Leon M., Chavez L., Surani S.: Hepatic hemangioma: What internists need to know. World J. Gastroenterol. 2020, 26, 11-20.

6. Lowe L. H., Marchant T. C., Rivard D. C., Scherbel A. J.: Vascular malformations: classification and terminology the radiologist needs to know. Seminar Roentgol. 2011, 66, 1208-1218.

7. Machado M. M., Rosa A. C. F., Lemes M. S., Milhomem da Mota O., Silva O. Ö., Olivier Campoli P. M., Filho J. B. S., Barreto P. A., Nunes R. A., Barreto M. C., Milhomem P. M., Milhomem L. M., Oliviera G. B., Oliviera F. B., Castro F. C. F., Brito A. M., Barros N., Cerri G. G.: Liver hemangioma: ultrasound and clinical features. Radiol. Bras. 2006, 39, 441-446.

8. Mavilla M. G., Pakala T., Molina M., Wu G. Y.: Differentiating cysts liver lesions: a review of imaging modalities, diagnosis and management. J. Clin. Transl. Hepatol. 2018, 6, 208-216.

9. Specchi S., Rossi F., Weise Ch., Morbito S., Petrovich N. P., Drees R., Thierry F., Ricciardi M., Penchome R., Areminise A., Pey P., Peak M., Panopoulos I., Nicoli S., Schwarz T.: Canine and feline abdominal arterioportal communications can be classified based on branching patterns in computed tomographic angiography. Vet. Radiol. Ultrasound 2018, 59, 687-696.

10. Szatmari V., Nemeth T., Kotai I., Voros L., Sotonyi P.: Doppler ultrasonographic diagnosis and anatomy of congenital intrahepatic arterioportal fistula in a puppy. Vet. Radiol. Ultrasound 2000, 41, 284-286.

11. Vilgrain V., Boulos L., Vulierme M. P., Denys A., Terris B., Menu Y.: Imaging of atypical hemangiomas of liver with pathologic correlation. RadioGraphics 2000, 20, 379-397.

12.Zwingenberger A. L., McLear R. C., Weisse C.: Diagnosis of arterioportal fistulae in four dogs using computed tomographic angiography. Vet. Radiol. Ultrasound 2005, 46, 472-477.

13. Zwingenberger A. L., Schwarz T.: Dual-phase CT angiography of the canine portal and hepatic vasculature. Vet. Radiol. Ultrasound 2004, 45, 117-124.

Corresponding author: dr n. wet. Anna Lojszczyk, Glęboka 30, 20-612 Lublin, Poland; e-mail: anna.lojszczyk@gmail.com 